\title{
A catalogue of bacterial swarm behaviour
}

Even under adverse conditions such as starvation, some bacteria can efficiently expand and move their colonies by rapidly migrating en masse, a process known as swarming. Although bacterial colonies have been studied by physicists for years, there is not yet a complete picture of how the physical properties of the cells, such as their shape, govern the behaviour of the swarm. Now, writing in Communications Physics, Avraham Be'er and colleagues fill in another piece of the puzzle, cataloguing the swarming behaviour of the rod-shaped Bacillus subtilis, as a function of the density of the colony and the aspect ratio of the cells.

B. subtilis is a bacterium found in soil and the gut of humans and animals. It is well-studied and the length of its cells can be controlled by known genetic manipulations. This controllability enabled Be'er et al. to select between four cell aspect ratios for their colonies. They recorded the motion of the colonies on a surface for a range of colony densities. Very sparse colonies of $B$. subtilis do not move, whereas very dense colonies are jammed. However, for intermediate densities, cell aspect ratio plays a role in the colony's behaviour. Longer cells form high-density clusters of moving cells that are separated by low-density regions containing only immobile cells (upper left panel of figure). If the mean density of the colony is large enough, then the moving clusters span the whole field of view of the experiment (upper right panel of figure). Comparison with earlier studies

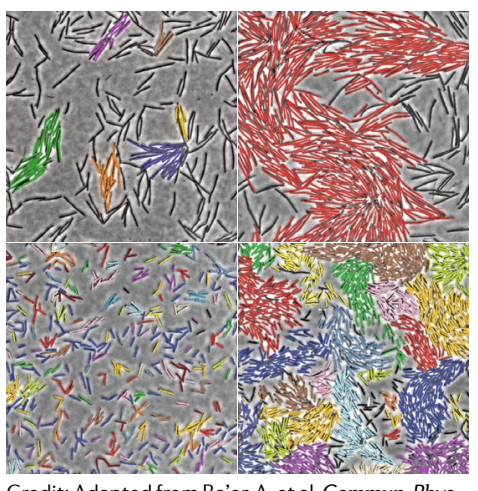

Credit: Adapted from Be'er, A. et al. Commun. Phys. 3, 66 (2020), CC BY 4.0

of similar self-propelled rods with known interactions indicates that the bacterial behaviour is dominated by short-range interactions. In contrast to long cells, shorter cells swarm in clusters that have uniform density across the surface (lower panels of figure). Be'er et al. posit that this uniform density arises because long-range hydrodynamic interactions suppress inhomogeneities.

Zoe Budrikis

ORIGINAL ARTICLE Be'er, A. et al. A phase diagram for bacterial swarming. Commun. Phys. 3, 66 (2020) 